\title{
LATERAL ELECTRON TRANSPORT THROUGH A QUANTUM DOT: COULOMB BLOCKADE AND QUANTUM TRANSPORT
}

J.G. Williamson(1,2), A.A.M. Staring(1), L.P. Kouwenhoven(3), H.van Houten(1), C.W.J. Beenakker(1), C.E. Timmering(1), M. Mabesoone(1),C.T. Foxon(4,5)

(1) Philips Research Laboratories,5600 JA Eindhoven, The Netherlands

(2) Now at the University of Glasgow Dept of Electronics and Electrical Engineering, Glasgow G12 800, U.K.

(3) Delft University of Technology, Faculty of Physics, 2600 GA Delft, The Netherlands.

(4) Philips Research Laboratories, Redhill RH1 5HA, U.K.

(5) Now at the University of Nottingham, Nottingham NG7 2RD, U.K..

We have fabricated a versatile quantum dot device,defined in a twodimensional electron gas by three pairs of gates. In the quantum transport regime, where the entrance and exit barrier conductances were greater than $2 \mathrm{e}^{2} / \mathrm{h}$, corresponding to two quantum point contacts in series, we have observed conductance plateaux corresponding to the lowest conductance point contact, modulated, at low temperature, by a period similar to that of the conductance plateau separation for a single quantum point contact. For the case where the conductance of both barriers was less than $2 \mathrm{e}^{2} / \mathrm{h}$, periodic oscillations have been observed as a function of gate voltage which we interpret as coulomb blockade oscillations. These oscillations were observed even when the electrons in the dot were poorly confined.

\section{INTRODUCTION}

Fabrication techniques for the lateral confinement of the two-dimensional electron gas in a GaAs-AlGaAs heterostructure have now advanced to the stage where it is possible to make controllable semiconductor devices which are sensitive to the effects of a single electron. In this paper we will discuss a quantum dot device where both the size of the dot, and the tunneling probabilities through the entrance and exit barriers, could be varied continuously. With this device it was possible to progress smoothly from the regime of quantum transport where the conductance was quantised $(1,2)$, to the regime of coulomb 
blockade of a single electron, which was, until recently, only available in metal systems (3). The first of these cases corresponds to two quantum point contacts in series which was first investigated experimentally by Wharam et al(4). The conductance was found to be enhanced above the value expected for two conductances in series. Beenakker and van Houten(5)have interpreted this behaviour as direct ballistic transmission from one point contact to another, enhanced by the typically horn-like nature of the electrostatic potential formed by a split-gate quantum point contact. This horn collimation effect has recently been demonstrated experimentally $(6)$. When the conductance of the two point-contacts, and of the device as a whole, falls below $2 \mathrm{e}^{2} / \mathrm{h}$ a new regime is entered, corresponding to electrons tunneling into and out of a quantum dot. Periodic oscillations were first observed in this regime by Scott-Thomas et al( 7$)$, in a narrow $S i$ inversion channel and interpreted in terms of a charge density wave $(8,9)$. Since then such oscillations have also been observed by several groups (10-13) in the conductance of a quantum dot, where a more natural explanation for their origin is the coulomb-blockade of tunneling $(3,14-17)$. It has also proved possible to extract the underlying zero-dimensional subband structure in such a device (18). Here we will be concerned with the transition region, where the tunneling barriers do not confine electrons strongly in the quantum dot, and will show that the oscillations persist into this regime.

\section{DEVICE DESCRIPTION}

The GaAs-AlGaAs heterostructure material used in this experiment contained a two-dimensional electron gas of carrier density of $2 \times 10^{11} \mathrm{~cm}^{-2}$ and a mobility of $2 \times 10^{6} \mathrm{~cm}^{2} / \mathrm{Vs}$, at low temperatures. A scanning electron micrograph of the gold gates defining the quantum dot is shown in Fig. 1.

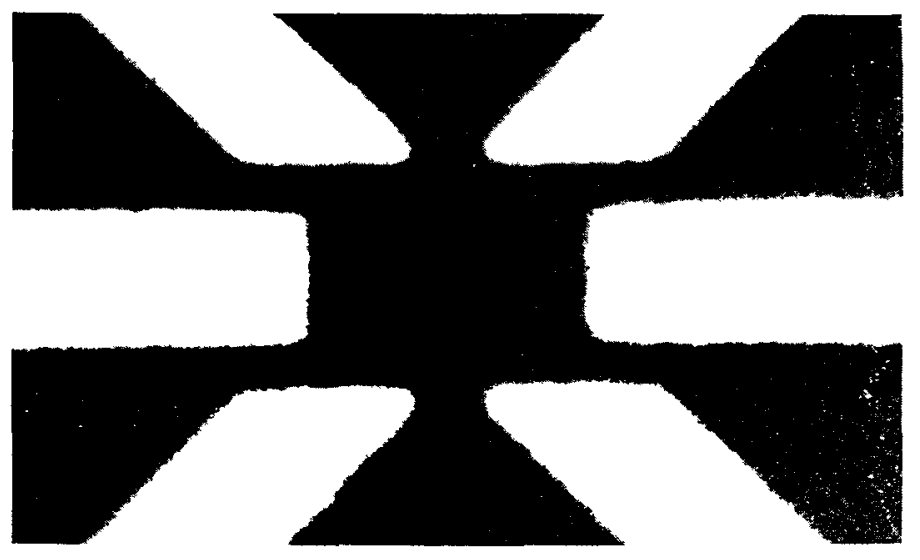

Figure 1: Scanning electron micrograph of the gates defining the quantum dot

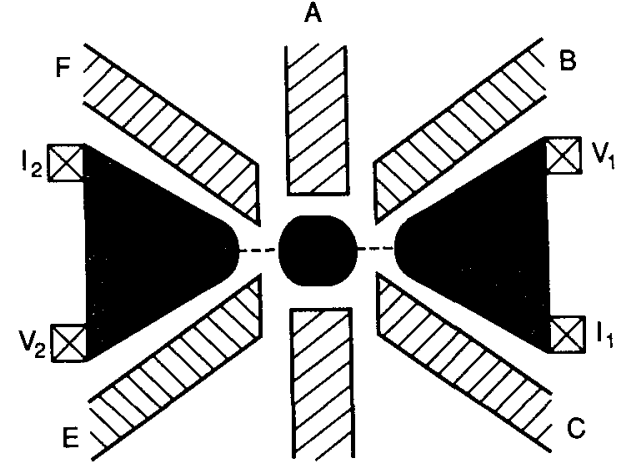

D

Figure 2: Schematic diagram of the quantum dot. Gates BC and EF define quantum point contacts which act as variable transparency tunnel barriers. Gates $A$ and D primarily effect the dot size. The current and voltage probes and magnetic field direction are also indicated.

Fig. 2 is a schematic diagram of the device formed in the plane of the twodimensional electron gas under a negative gate bias of, typically, $-0.7 \mathrm{~V}$. The adjustable tunnel barriers are quantum point contacts formed by the top and bottom pairs of gates EF and BC. The quantum dot size and number of confined electrons may be controlled using the two side gates A and D. For all data presented here the device is as shown in Fig. 2, in that there were two, and only two channels between the quantum dot and the broad two-dimensional electron gas regions, and not, for example, any connection through to the region between gates $\mathrm{A}$ and $\mathrm{B}$. The lithographic quantum point contact separation BC was 0.3 $\mu \mathrm{m}$, the separation between the pairs BF was $0.7 \mu \mathrm{m}$ and the side gate spacing $\mathrm{AD}$ was $0.9 \mu \mathrm{m}$. The layer structure was $17 \mathrm{~nm} \mathrm{GaAs}$ cap, $39 \mathrm{~nm}$ of $1.33 \times$ $1018 \mathrm{~cm}^{3}$ Si doped AlGaAs, followed by $40 \mathrm{~nm}$ of undoped AlGaAs, followed by a GaAs buffer. Note that the side gates $\mathrm{AD}$ affect the potential in the regions under the point contact gates BCEF and vice-versa. These effects cannot be avioded, but for this particular device were relatively small as we will discuss. The conductance measurements were carried out using an $\mathrm{AC}$ lock-in technique with an excitation of, typically, $10 \mu \mathrm{V}$. All measurements were carried out at $100 \mathrm{mK}$ unless otherwise stated.The diagonal measurement configuration used (illustrated in Fig. 1) yields an effective two-terminal conductance even in the presence of a magnetic field(19). 


\section{EXPERIMENT}

This device was extremely versatile in that the six independent gates allowed us to vary both the dot size and tunnel barrier transparency. Unfortunately this also means that there are many parameters, not all of them independent, which one may vary. To save space we have chosen to present some data in the form of three dimensional plots which summarise many of the main features of the experiment at the expense of a certain amount of clarity. We will first discuss the effect of varying the gate voltages defining the entrance and exit point contacts, at zero magnetic field, and for different temperatures (the quantum ballistic regime). We will then concentrate on two particular gate voltages, corresponding to tunneling to and from the quantum dot (the coulomb blockade regime), and invesitigate the device behaviour as a function of the dot size (gates A and D) and the magnetic field.

A 3-dimensional plot of the two terminal device conductance as a function of the entrance and exit quantum point contact potential at $1.2 \mathrm{~K}$ is presented in Fig. 3. A vertical axis is given to set the general scale, but this is only really useful

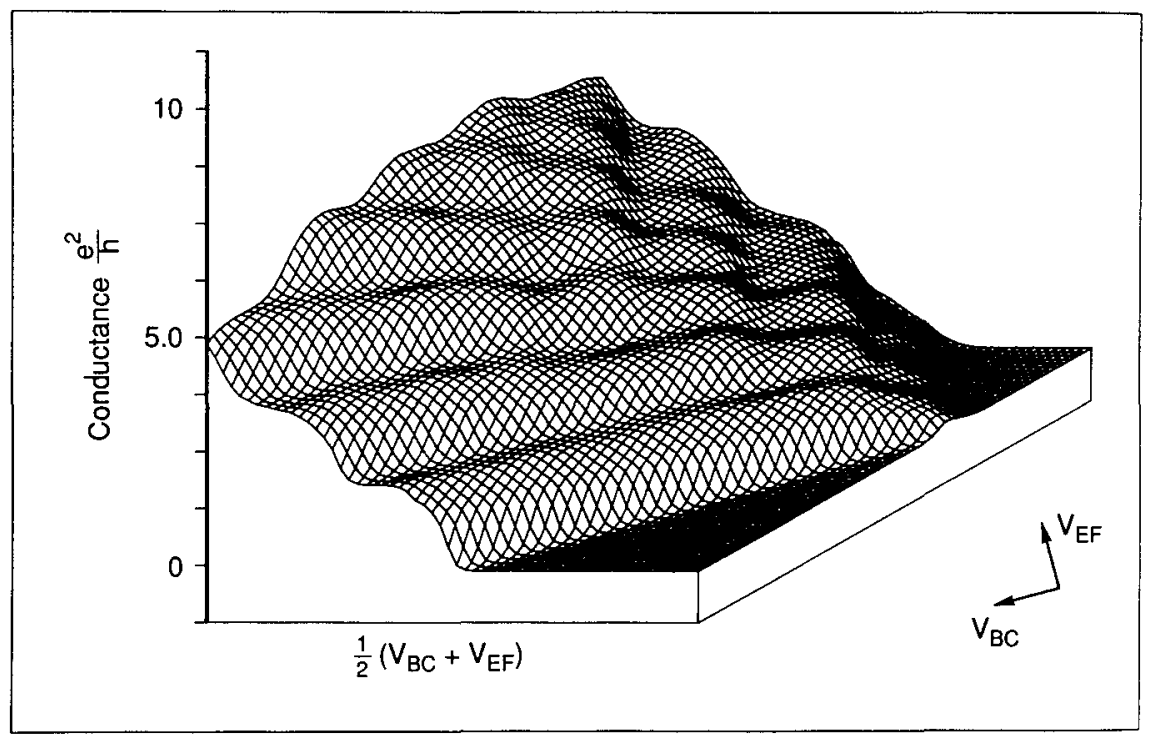

Figure 3: Conductance versus gate voltage on entrance and exit quantum point contact gates $\mathrm{EF}$ and $\mathrm{BC}$ for $\mathrm{T}=1.2 \mathrm{~K}$ and $\mathrm{B}=0 \mathrm{~T}$. The horizontal axis corresponds to $1 / 2\left(\mathrm{~V}_{\mathrm{bc}}+\mathrm{V}_{\mathrm{ef}}\right)$ running from $-0.5 \mathrm{~V}$ to $-0.8 \mathrm{~V}$. The inclined axis corresponds to a change in $1 / 2\left(\mathrm{~V}_{\mathrm{bc}}-\mathrm{V}_{\mathrm{ef}}\right)$ by $0.3 \mathrm{~V}$. Lines of constant $\mathrm{V}_{\mathrm{bc}}$ are diagonal from front right to rear left, and constant $\mathrm{V}_{\mathrm{ef}}$ from front left to rear right. where this intersects the surface, and quantitative comparisons can better be made with reference to the contour plot in Fig. 5 . Well-defined plateaux are observed in the conductance.Any cross-section of this plot yields a one dimensional plot of the conductance as a function of gate voltage similar to that observed for a single quantum point contact $(1,2)$.In particular, a diagonal cross section through the plot, in the direction of $V_{E F}$ or $V_{B C}$, as illustrated on the figure, corresponds to varying the gate voltage on one of the two series point contact gate pairs alone while keeping the other pair fixed. A striking feature of this plot is that, except for a central ridge region where both quantum point contacts are equally important, the conductance of the system as a whole is dominated by one or other of the quantum point contacts. This is evidenced by the triangular ridges visible in the plot, where, in most regions, the rate of change of the conductance with respect to the gate voltage on the lower conductance point contact is very much larger than that with respect to the other point contact. A comparison with a simple model suggests that this effect is stronger than one would expect for simple ohmic addition alone suggesting that ballistic transport plays an important role here. This is supported by the fact that the conductance of the lowest plateau, near to the ridge where both point contacts have equal importance, is significantly greater than $\mathrm{e}^{2} / \mathrm{h}$. It may also be concluded that the potential under one pair of gates is only weakly effected by the gate voltage on the other pair, since otherwise the conductance in a region dominated by one point contact would be influenced by the fringe-field of the other. Note that, while the plateaux correspond to an integer number of one-dimensional subbands being occupied in the lowest conductance quantum point contact, the conductances are always lower than that integer times $2 \mathrm{e}^{2} / \mathrm{h}$. This may be due the effects of the second,series point contact, but it should be borne in mind that, even for single quantum point contacts, a well-defined plateau may be present at conductances lower than the quantised values, due to non-integral transmission probabilities $(20)$, and this may also play a role here as no "background" resistance subtraction has been applied. We note also that the central ridge does not correspond to the having the same voltage on both sets of gates, since both quantum point contacts do not cut-off at the same voltage. The equal voltage axis is parallel to the horizontal axis and roughly two thirds of the way from the front. The effect of reducing the lemperature to $100 \mathrm{mK}$ is illustrated in figure 4 . The extra structure seen was found to be extremely reproducible over a period of several days. This structure appears to be quasi-periodic with a gate voltage scale similar to that of the plateau spacing. The same data is presented as a contour plot in Fig. 5. The contour interval is $0.2 \mathrm{e}^{2} / \mathrm{h}$. Note again the dominant triangular structure, indicative of ballistic transport dominated by the lowest conductance point contact, and that the conductance where both point contacts have one subband occupied is about 1.4 $\mathrm{c}^{2} / \mathrm{h}$. The ridges of sharp increase in the conduction correspond to a new onedimensional sub-band entering the dominant quantum point contact. The extrapolation of these ridges to the case where the other point contact is dominant seems to suggest that at these intersections the conductance is, in general, closer 10) the nominal quantised conductance. Note that we speculate here based on an extremely small data sample consisting of a handful of intersections for a single device.If this effect were confirmed, however, this may suggest that 


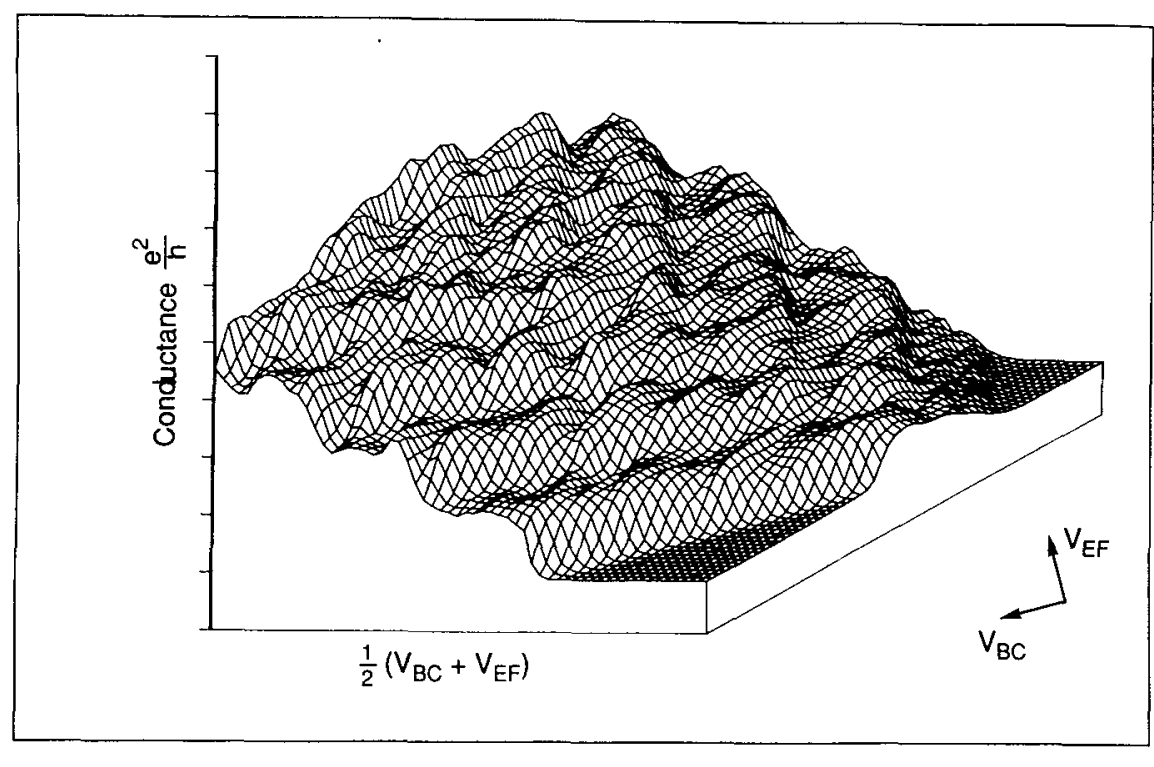

Figure 4: As Fig. 3 but $T=100 \mathrm{mK}, 1 / 2\left(\mathrm{~V}_{\mathrm{bc}}+\mathrm{V}_{\mathrm{ef}}\right)$ from $-0.45 \mathrm{~V}$ to $-0.75 \mathrm{~V}$

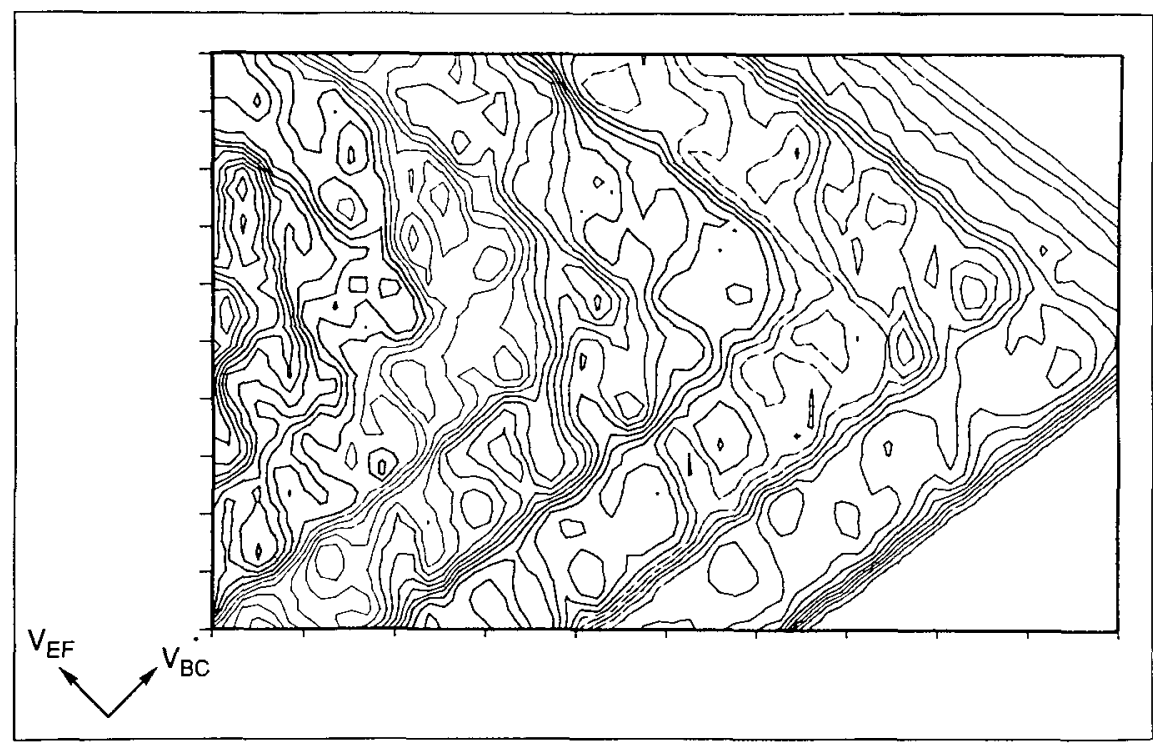

Figure 5: Contour plot of Fig. 4 with contour interval $0.2 \mathrm{e}^{2 / \mathrm{h}} .1 / 2\left(\mathrm{~V}_{\mathrm{bc}}+\right.$ $\mathrm{V}_{\mathrm{ef}}$ ) from $-0.77 \mathrm{~V}$ to $-0.83 \mathrm{~V}$. The directions of $\mathrm{V}_{\mathrm{bc}}$ and $\mathrm{V}_{\mathrm{ef}}$ are indicated.

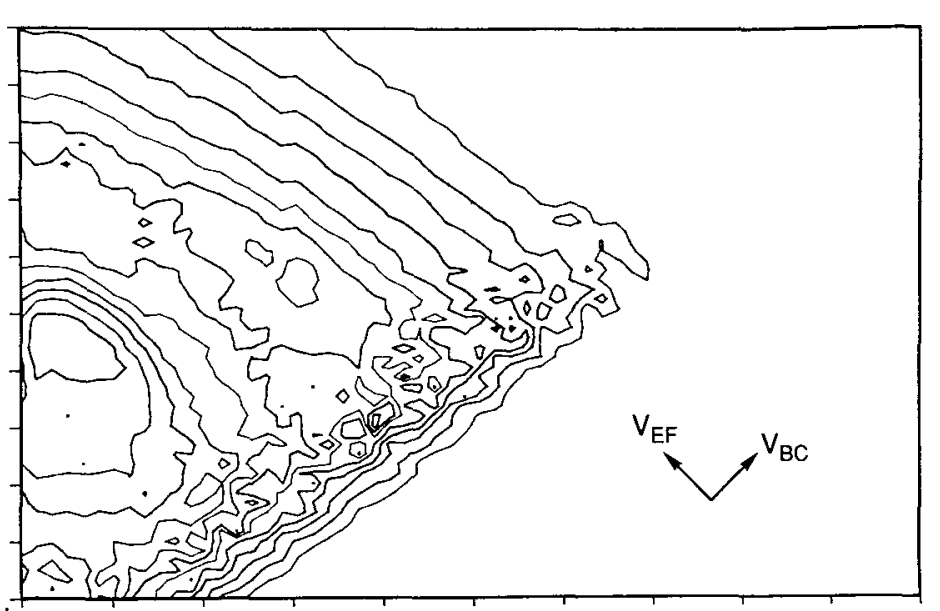

Figure 6: Similar to Fig. 5 but higher resolution, and containing the regime where periodic oscillations have been observed. The horizontal axis corresponds to an increase of the potential on all four gates simultaneously by $0.06 \mathrm{~V}$. The vertical axis corresponds to a change in the difference by $0.12 \mathrm{~V}$ The contour interval is $0.55 \mathrm{e}^{2 / \mathrm{h}}$

backscattering is reduced close to the point where a new subband were entered. At this point the one-dimensional density of states is maximal, and it is possible that the probability of an individual electron matching both energy and momentum across the two point contacts is enhanced due to the increased density of states. It is equally likely, however, that the extra structure observed is simply due to quantum interference between the entrance and exit quantum point contacts. Fig. 6 shows a close-up of the range missing in Fig. 5, for the region where the two quantum point contacts are in the tunneling regime of conductance less than $2 \mathrm{e}^{2} / \mathrm{h}$. It is surprising that, even in this regime, one or other of the two point contacts appears to dominate the conductance of the device as a whole. Even though one is firmly in the tunneling regime, this aspect of transport still appears to be ballistic. The peak visible in the lower left corner, however, shows that other factors in the underlying device potential landscape may play a role here $(20)$. This, and the fact that this effect has only been observed in a single device, mean that it is premature to draw firm conclusions at this stage, and that more work is required to verify this effect. 
We have seen that, in this particular device, we may set the relative importance of the two point contacts with a great deal of freedom. We now exploit this to fix $V_{b c}$ and $V_{\text {ef }}$ such that both are in the tunneling regime of conductance less than $2 \mathrm{e}^{2} / \mathrm{h}$, corresponding to the ridge of Figs. 45 and 6 . We vary the size of the quantum dot using the control gates $\mathrm{AD}$, for various values of magnetic field perpendicular to the plane of the two-dimensional electron gas. Fig. 7 shows six such plots for magnetic fields between 0 and $3.75 \mathrm{~T}$ with subsequent curves offset by one scale division, where Fig. 8 shows the extreme values with no offset. These data will also be presented elsewhere(12). Clear, periodic, oscillations are observed, which increase in magnitude with increasing magnetic field. Such oscillations were observed for all of the region corresponding to Fig. 6 , but the oscillations were strongest when both quantum point contacts had equal conductance. The extra structure visible as small peaks in Fig. 6 is probably due to these oscillations, but then poorly resolved due the the large gate voltage step size (1/60 of the axis).

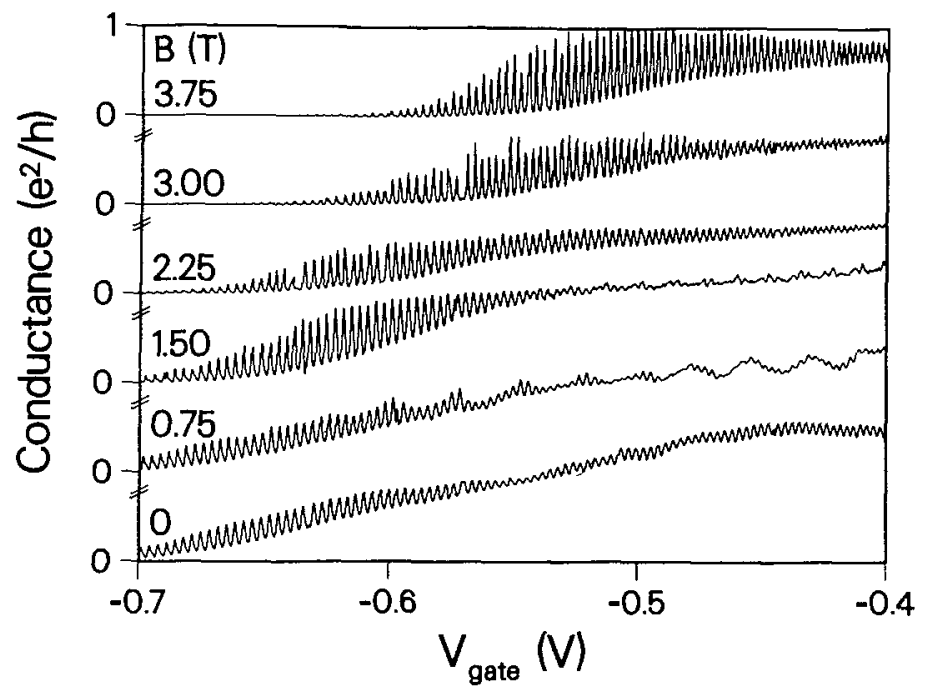

Figure 7 Quantum dot conductance versus control gate voltage $V_{\text {ad }}$ for six magnetic field values. $V_{b c}$ and $V_{e f}$ were fixed such that the conductance of each point contact at zero field was less than $2 e^{2} / \mathrm{h}$. Successive curves have been offset by $\mathrm{e}^{2} / \mathrm{h}$.

The oscillation period increases slowly with increasing negative gate voltage This may be attributed to the decrease in the size of the dot, and hence the mutual capacitance from it to the control gates. The background conductance increases with decreasing negative control gate voltage. This may be due to the action of the fringe-fields of the control gates on the transparency of the tunnel barriers. The magnetic field has almost no effect on the period (less than a few percent), and no

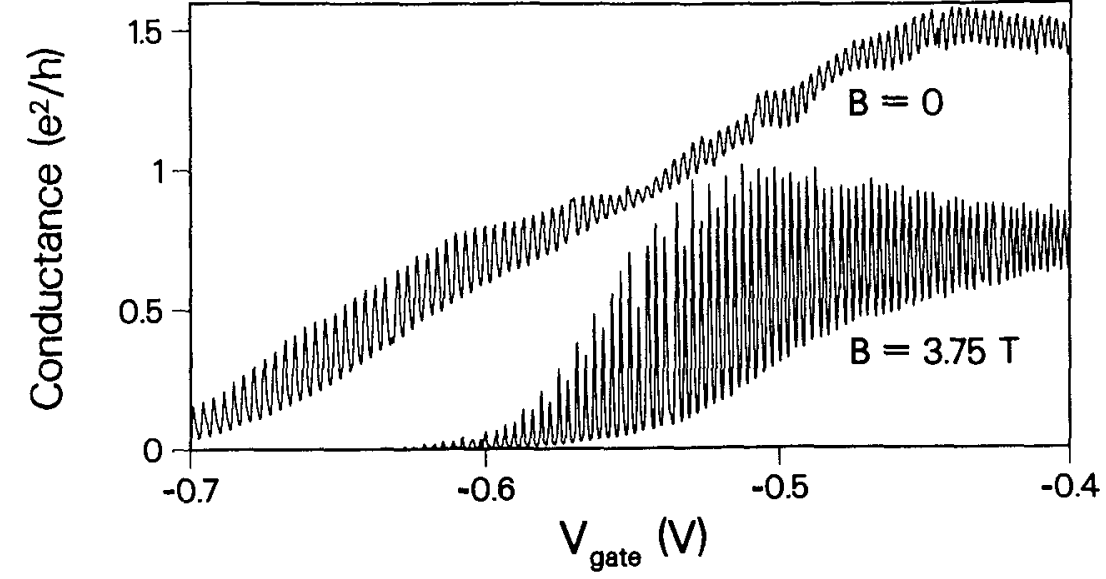

Figure 8: Detail of data from fig. 7. Illustrating the extra modulation of the conductance peaks at $3.75 \mathrm{~T}$ and the independance of the oscillation period over a wide range of magnetic field.

spin-splitting of the conductance peaks is observed. The gate voltage at which the total device conductance approaches zero, increases monotonically with the magnetic field. This may be attributed to a magnetic depopulation of the quantum dot. An additional slow modulation with period roughly $26 \mathrm{mV}$ at $0.75 \mathrm{~T}$ and 13 ${ }_{\mathrm{HI}} \mathrm{V}$ at $1.5 \mathrm{~T}$ is observed for gate voltages between -0.4 and $-0.55 \mathrm{~V}$. At $\mathrm{B}=3.75$ I a much faster modulation with twice the fundamental period is observed(see I g. 8). These effects may be due to energy and momentum matching of the vates at the entrance and exit of the dot and in the dot itself enhancing or uuppressing the total tunneling rate. The peak-to-valley amplitude of the "rcillations is always less than $\mathrm{e} 2 / \mathrm{h}$, though the peaks, at $\mathrm{B}=0 \mathrm{~T}$ exceed this value. The conductance minima in Fig. 8 are not exponentially suppressed, even though the temperatures are low.Many of the features of our data are welldescribed by a model of the coulomb blockade of tunneling $(16,17)$ we review hriefly our evidence for this. No oscillations were observed when either of the puint contacts had conductance greater than $2 \mathrm{e}^{2} / \mathrm{h}(21)$. No magnetoconductance incillations were observed.We estimate the dot area as $0.4 \times 0.6 \mu \mathrm{m}^{2}$. From the I ermi energy and the two dimensional density of states we then extract an upper limit on the subband energy spacing of $30 \mu \mathrm{eV}$. We observe oscillations up to 1) $9 \mathrm{~K}$, which implies an activation energy of $4 \mathrm{k}_{\mathrm{B}} \mathrm{T} \approx 0.3 \mathrm{meV}$. An explanation in terms of resonant tunneling of non-interacting electrons is therefore excluded. The charging energy of a flat disc of diameter $d=0.6 \mu \mathrm{m}$ is $\mathrm{e}^{2 / 4} \varepsilon_{0} \varepsilon_{\mathrm{r}} \mathrm{d} \approx 0.5 \mathrm{meV}$ lor $\varepsilon_{\mathrm{r}}=13$, which explains the large activation energy of the oscillations. The wcillation period is consistent with a gate-dot capacitance of $5 \times 10^{-17} \mathrm{~F}$, which 
is not unreasonable. We conclude then that many experimental features are indeed consistent with being due to the coulomb-blockade of tunneling. Fig. 9 shows one of the transition regions of the onset of the oscillations. The top curve (not offset) corresponds to both point contacts having a conductance just below $2 \mathrm{e}^{2} \mathrm{~h}$ The total device conductance is also of this order. Subsequent curves correspond to reducing the gate voltage on one of the two point contacts by $0.01 \mathrm{~V}$. This is observed to move the point of onset of the oscillations in the control gate voltag by roughly $0.1 \mathrm{~V}$, implying that the control gates have roughly a tenth of the effect of the point contact gates on the point contact potential. This is consistent with the relative gate voltages required to pinch the device off using the side gates (Fig.8) or the point contact gates (Fig 5,6). This experimental independence of the various gates from each other is quite remarkable. To check if the loca electrostatic potential landscape for this particular device contained any strong fluctations we performed an experiment where we moved the whole quantum do sideways $(20)$, this suggested that, in this particular device, the quantum dot may have been enhanced by a natural potential fluctuation close to the dot centre(22), which may account for the exceptional controlability of this particula device.

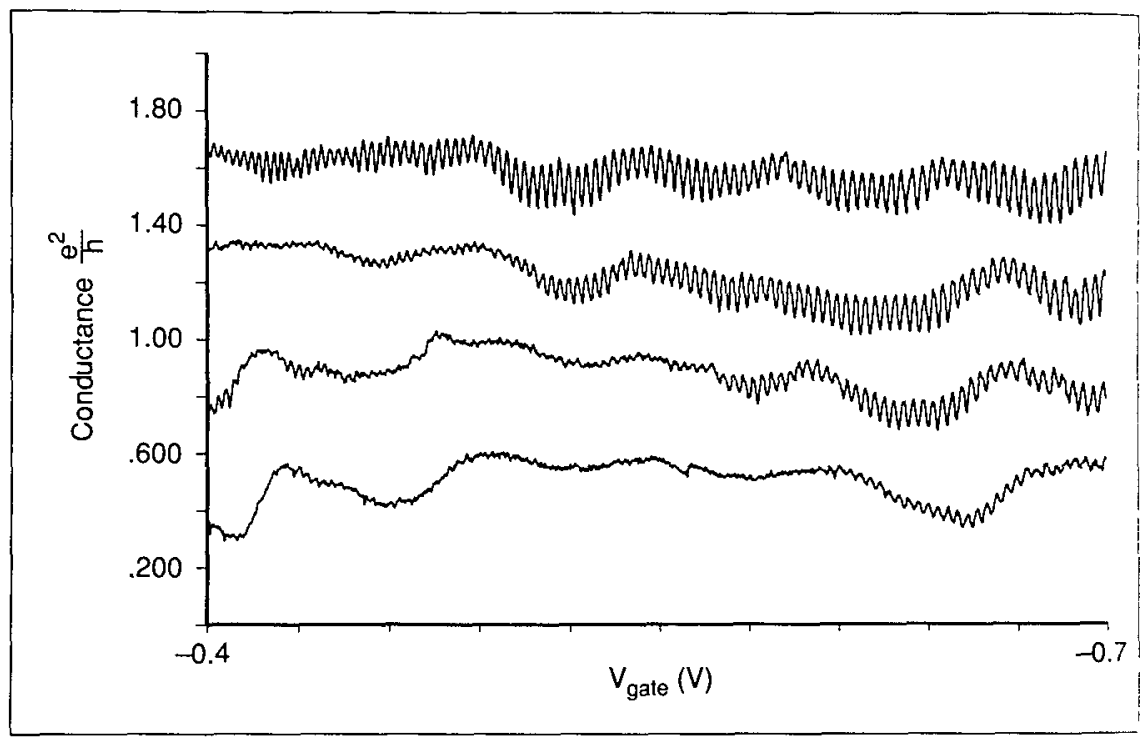

Figure 9: Onset of oscillations for zero magnetic field around the region where one of the two point contacts enters the lowest conductance plateau illustrated in Fig. 3. The top curve, which is not offset, shows fullydeveloped oscillations in the region where the conductance of both poin contacts, and the device as a whole, is of the order of $2 \mathrm{e}^{2} / \mathrm{h}$. Subsequent curves show the effect of decreasing the voltage on one set of point-contact gates in $0.01 \mathrm{~V}$ steps. The curves, which would otherwise lie on top of each other, have been offset for clarity.
We conclude that even where the transparency of the tunnel barriers is relatively high (conductance of the order of $2 \mathrm{e}^{2} / \mathrm{h}$ ) so that the quantum dot is barely defined, coulomb-blockade oscillations are present. In this regime the electrons "ooze" into and out of the dot, rather than being strongly and definitely confined in ${ }_{11}(23)$. Strictly speaking, it is not appropriate to talk about coulomb blockade in this region, though the data suggests that the oscillations here have the same physical origin as those in the more strongly confined case illustrated in Figs. 7 phys . and 8. This regime then, lies outside that addressed by the standard theories $(3)$. Virtual tunneling may play a role here $(24,25)$, though at present, these theories do not well describe the regime of interest here(26). For this regime the pinned harge density wave picture seems more appropriate $(7-9)$, but there is a smooth transition of the oscillations to the regime of conductance much lower than $\mathrm{e}^{2 / \mathrm{h}}$ where an explanation in terms of tunneling to and from a quantum dot with well defined capacitance seems more natural, and it is hard to believe that different physical mechanisms play a role in these two regimes. To make progress here it may be fruitful to consider further the nature of the localisation of charge in solid tate systems $(27)$

\section{CONCLUSIONS}

We have used the flexibility of this device to investigate several different regimes of electron transport. We have observed quasi-periodic structure on the plateaux of two quantum point contacts in series. We interpret this as being due ther to a density of states effect, or to quantum interference. In the tunneling regime periodic oscillations have been observed, which are strongly enhanced in a magnetic field up to a peak to valley amplitude of $\mathrm{e}^{2 / \mathrm{h}}$. We interpret these as coulomb blockade oscillations. These persisted into the region where the total device, and the individual point contact conductances were of the order of the last quantised plateau conductance.

\section{ACKNOWLEDGEMENTS}

It is a pleasure to acknowledge valuable discussions with Bruce Alphenaar, Martin van der Mark, Charlie Johnson, Neil Johnson, Mark Reed and Frank Stern and the support of Martin Schuurmans. This work was partly funded under ESPRIT basic research action 3133.

\section{REFERENCES}

1. B.J. van Wees, H. van Houten, C.W.J. Beenakker, J.G. Williamson, L.P. Kouwenhoven, D. van der Marel, and C.T. Foxon, Phys. Rev. Lett. 24 848 (1988).

2. D.A Wharam, T.J. Thornton, R. Newbury, M. Pepper, H. Ahmed, J.E.F. Frost, D.G. Hasko, D.C. Peacock, D.A. Ritchie, and G.A.C. Jones, J. Phys. C 21, L209 (1988).

3. For a review see K.K. Likharev, IBM J. Res. Dev. 32, 144 (1988). 
4. D.A. Wharam, M. Pepper, H. Ahmed, J.E.F. Frost, D.G. Hasko, D.C. Peacock, D.A. Ritchie, and G.A.C. Jones, J.Phys. C 21, L887 (1988)

5. C.W.J. Beenakker and H. van Houten, Phys. Rev. B 39, 10445 (1989).

6. L.W. Molenkamp, A.A.M. Staring, C.W.J. Beenakker, R. Eppenga, C.E. Timmering and J.G. Williamson, Phys. Rev. B, 41, 1274 (1990).

7. J.H.F. Scott-Thomas, S.B. Field, M.A. Kastner, H.I. Smith, and D.A. Antoniadas, Phys Rev. Lett. 62, 583 (1989).

8. A.I. Larkin and P.A. Lee, Phys. Rev. B 17, 1596 (1978).

9. P.A. Lee and T.M. Rice, Phys. Rev. B 19, 3970 (1979).

10 U. Meirav, M.A. Kastner, and S.J. Wind, Phys. Rev. Lett. 65,771 (1990).

11. L.P. Kouwenhoven, N.C. van der Vaart, A.T. Johnson, C.J.P.M. Harmans, J.G. Williamson, A.A.M. Staring, and C.T. Foxon, Festkorperforschung / Advances in Solid state Physics (Vol. 31, to be published).

12. A.A.M. Staring, J.G. Williamson, H. van Houten, C.W.J. Beenakker, L.P.Kouwenhoven, and C.T. Foxon, Proc. Int. Symp. on Analogies in Optics and micro-electronics, D. Lenstra, and W. van Haeringen, eds. Physica B andC (North-Holland, Amsterdam, to be published).

13. C.J.B. Ford, Physica Scripta, to be published.

14. L.I. Glazman and R.I. Shekhter, J. Phys. Condens. Matter 15811 (1989).

15. H. van Houten and C.W.J. Beenakker, Phys. Rev. Lett. 63, 1893 (1989).

16. C.W.J. Beenakker, Phys. Rev. B 44, 1646, (1991).

17. Y. Meir, N.S. Wingreen, and P.A. Lee, Phys. Rev. Lett. 66, 3048 (1991).

18. P.L. McEuen, E.B. Foxman, U. Meirav, M.A. Kastner, Y. Meir, N.S Wingreen and S.J. Wind Phys. Rev. Lett. 66, 1926 (1991).

19. For a review see C.W.J. Beenakker and H. van Houten, Solid State Physics 44, 1 (1991).

20. J.G. Williamson, C.E. Timmering, C.J.P.M. Harmans, J.J. Harris and C.T. Foxon, Phys. Rev. B 42, 7675 (1990).

21. See also: L.P. Kouwenhoven, N.C. van der Vaart, W. Kool, C.J.P.M. Harmans, J.G. Williamson, A.A.M. Staring, and C.T. Foxon, to be published.

22. J. Nixon and J. Davies, Phys. Rev. B 41, 7929 (1990).

23. C.S. Lent, these proceedings.

24. D.V. Averin and A.A. Odintsov, Phys Lett. A 140, 251 (1989);

25. L.I. Glazman and K.A. Matveev, Pis'ma Zh. Eksp. Teor. Fiz. 51, 425 (1990) [JETP Lett. 51, 484 (1990)].

26. D.V. Averin, private communication

27. A.S. Goldhaber and S.A. Kivelson, Phys. Lett. B 256, 245 (1991).

\section{QUANTIZED TRANSPORT THROUGH A QUANTUM DOT TURNSTILE}

A.T. Johnson, L.P. Kouwenhoven, N.C. van der Vaart, and C.J.P.M. Harmans

$$
\text { Faculty of Applied Physics, Delft University of Technology }
$$
P.O. Box 5046, 2600 GA Delft, The Netherlands

\section{C.T. Foxon}

Philips Research Laboratories, Redhill, Surrey RH15HA, United Kingdom

We have observed a quantized current in a lateral quantum dot, defined by metal gates in the two-dimensional electron gas (2DEG) of a GaAs-AlGaAs heterostructure. We create oscillating tunnel barriers in the 2DEG with two RF ugnals, which in combination with the Coulomb blockade of tunneling produce current plateaus in the I-V curves at integer multiples of ef, $f$ being the $R F$ irequency. This shows that the number of electrons that pass through the quantum Jot each cycle is an integer.

\section{INTRODUCTION}

Electron transport through submicron systems at low temperature can be regulated by the electrostatic energy needed to charge a small structure by a single ilectron. This was first recognized in granular metal samples ${ }^{1}$ and enjoys a rcvival of interest with the widespread availability of electron-beam lithography. ${ }^{2}$ Fulton and Dolan ${ }^{3}$ controlled the transport-limiting energy, and therefore the current through two tunnel junctions in series, by a gate voltage capacitively coupled to the small center electrode. Delsing et al. ${ }^{4}$ locked the tunneling of ungle electrons through a 1-d array of junctions to an external RF signal. (icerligs et al. ${ }^{5}$ coupled RF to the center island of a sample of 4 junctions in wries. They modulated the Coulomb gap so that precisely one electron per cycle passed through their electron turnstile device. This control of current at the ingle-electron level has both fundamental interest and profound device implications. It is natural to ask whether it can be extended to non-metal systems, whose properties allow possibilities not present in metal tunnel junctions.

Here we present measurements on a quantum dot turnstile (QDT), defined by mctal gates in a two-dimensional electron gas (2DEG). ${ }^{6}$ The quantum dot is 